\title{
Investigation on the Horizontal Landing Velocity and Pitch Angle Impact on the Soft-Landing Dynamic Characteristics
}

\author{
Qing Lin $\mathbb{D}^{1}$ and Jie Ren ${ }^{2}$ \\ ${ }^{1}$ Aerospace System Engineering Shanghai, Shanghai 201109, China \\ ${ }^{2}$ Honeywell Integrated Technology (China) Co., Ltd., Shanghai 201203, China \\ Correspondence should be addressed to Qing Lin; 1784238@qq.com
}

Received 18 August 2021; Revised 28 September 2021; Accepted 6 December 2021; Published 25 January 2022

Academic Editor: Yue Wang

Copyright (C) 2022 Qing Lin and Jie Ren. This is an open access article distributed under the Creative Commons Attribution License, which permits unrestricted use, distribution, and reproduction in any medium, provided the original work is properly cited.

\begin{abstract}
The dynamic analysis of the soft landing of the lunar probe is very important to the design of the probe. The initial movement and attitude parameters of the probe during landing have a direct influence on the landing impact. In order to investigate the lunar probe soft-landing dynamic impact by different initial horizontal velocities, pitch angles, and inclinations of the lunar slope, an inertial force-based 7-DOF soft-landing dynamic model is applied under two conditions: the upward and downward slope landing surfaces. The impact on the dynamic characteristics of soft landing is analyzed in terms of body displacement, body overload, and the forces of the primary and secondary buffer struts due to the change of initial horizontal velocity and initial pitch angle of the probe. The result shows that, in 2-2 landing mode, the stress conditions on the primary and secondary struts are obviously impacted by initial horizontal velocity, and the initial pitch angle affects the body overload and the loading state of the secondary buffer strut. The body overload and landing impact could be significantly mitigated if the lunar probe's horizontal landing speed is limited within $1 \mathrm{~m} / \mathrm{s}$, the pitch angle is limited within $12^{\circ}$, and the landing is along the uphill terrain with the inclination of the lunar slope less and equal to $9^{\circ}$. The analysis can directly determine the range of the horizontal speed and pitch attitude angle to ensure the safety of landing, and provide a reference for the reasonable control design of the lander's horizontal speed and pitch attitude.
\end{abstract}

\section{Introduction}

The soft-landing dynamics research is one of the most critical aspects in the development of the lunar lander. The landing impact process is simulated and analyzed to predict the dynamic characteristics of the lander. The soft-landing dynamics characteristics mainly focused on predicting the maximum acceleration response on the lander payload and the maximum force of the core components at different initial landing conditions, then determining the maximum expected mechanical environment and guiding the design of the main structure and buffer mechanism of the lunar lander. The initial horizontal landing velocity and initial landing pitch angle of the lander are two key landing condition factors, which have a great impact on the maximum acceleration response of the lander payload and the structural strength of the buffer mech- anism. Once the buffer mechanism loses balance on structural strength, the lander will tip over, which makes the whole mission fail [1].

The research on the soft-landing characteristics of the lunar lander can be divided into two stages. The first round of lunar exploration (1958-1979) is Apollo lunar exploration. A new global round of lunar exploration (1994-present) is Chang'e project. During Apollo's project, most of the softlanding dynamics studies established a simplified model of soft-landing dynamics to make rigid body dynamics of the lander and used vector mechanics or analytical mechanics to solve system dynamic and kinetic equations. Lavender simplified the lunar lander into a two-dimensional rigid body model [2]; based on which, the impacts of lunar inclination, friction coefficient, initial landing velocity, buffer crushing force, and number of landing legs on the landing stability of the lander 
were investigated [3]. He also summarized the previous lunar lander soft-landing dynamics analysis method and pointed out that the landing stability of the lander was greatly affected by the details of the dynamic model [4]. Both Black [5] and Walton et al. $[6,7]$ established a soft-landing dynamics model of the lunar lander to investigate landing stability. Zupp and Doiron [8] discussed the shortcomings of NASA's previous soft-landing dynamics model and established a more refined dynamic simulation model. During the new round of global lunar exploration, due to the fast development of computational mechanics, computational multibody system dynamics and finite element analysis techniques began to be applied to spacecraft dynamics analysis, especially the maturity of finite element technology, which provides conditions for the dynamic response analysis of soft landing. During this period, nonlinear finite element method is majorly used on the softlanding dynamics research. Large engineering simulation software is applied to establish an accurate three-dimensional model of the lunar lander to simulate and calculate the softlanding process. Wan Junlin [9] and Lin Qing [10] used the nonlinear finite element method to establish the soft landing dynamics model of the lander, and used the MSC. Dytran to calculate the soft-landing process after the flexible disposition of lunar lander and lunar soil. During the Apollo lunar exploration period, the soft-landing dynamics study considered the lander as a rigid body. The soft-landing dynamics simplified math model was used to analyze the rigid body dynamics. The main reason was the computing capability of the computer at that point was too low to handle complicated large models. In addition, the local response on the lander is not as important as landing stability while the landing stability analysis can be done by rigid body dynamics model with expected accuracy. Recently, the key point of soft-landing dynamics research moves to the landing impact response of large complex landers. The dynamics model is considerably closer to reality than in the past and can reflect the impact mechanics at different payload points. The nonlinear finite element modeling method needs to completely consider the influence of various nonlinear factors. In Reference [9], analysis of the one-leg landing condition of the lander 1/4 model takes 20 hours which indicates that the nonlinear finite element modeling method requires large amount of calculation and cannot effectively modify the parameters of the buffer mechanism and material in real time, which is contrary to the modern concept of rapid iterative design analysis.

Because of the deficiencies mentioned above, a previously built 2-2 landing mode soft-landing dynamic model of the lunar lander [11] is used in this analysis. Two landing conditions, uphill and downhill along the lunar surface, are investigated. The impacts of horizontal landing velocity, landing pitch angle, and the inclination of the lunar slope on the soft-landing dynamic characteristic are analyzed from the following perspectives: body displacement, body overload, and maximum forces on primary and secondary struts. The dynamic model contains 7 DOF, which is the least in the research of the lunar lander overall dynamics. It significantly reduces the complexity of model analysis and the difficulty of problem solving. The precision of the dynamic model is also verified by experiment.

\section{Soft-Landing Dynamic Model of Lunar Lander}

A previously built and verified 2-2 soft-landing dynamic model of the lunar lander [11] is used to investigate the impacts of horizontal landing velocity and landing pitch angle on the soft-landing dynamics. The following section introduces this soft-landing dynamic model.

Figure 1 is a schematic diagram of the force analysis of the entire lunar lander in the 2-2 symmetrical landing mode. Table 1 shows the definitions of force, angle, and key point of lunar lander force analysis in Figure 1.

As depicted in Figure 1, the lander is divided into three portions: the body part and two left-right symmetrical landing buffer struts (primary strut+secondary strut) and the foot pad. The body part is considered elastic, and the landing struts and foot pad are considered inelastic.

In the inertial coordinate system $O-X Y$, the dynamic equation in the vertical direction of the body part is as follows:

$$
m \ddot{y}=F_{m u 1} \sin \alpha_{m 1}+F_{m u 2} \sin \alpha_{m 2}-N_{m u 1} \cos \alpha_{m 1}-N_{m u 2} \cos \alpha_{m 2}+F_{s u 1} \sin \alpha_{s 1}+F_{s u 2} \sin \alpha_{s 2}-W+\left(T_{1}+T_{2}\right) \cos \varphi \text {. }
$$

The dynamic equation in the horizontal direction is as follows:

$$
m \ddot{x}=F_{m u 1} \cos \alpha_{m 1}-F_{m u 2} \cos \alpha_{m 2}+N_{m u 1} \sin \alpha_{m 1}-N_{m u 2} \sin \alpha_{m 2}+F_{s u 1} \cos \alpha_{s 1}-F_{s u 2} \cos \alpha_{s 2}-\left(T_{1}+T_{2}\right) \sin \varphi
$$




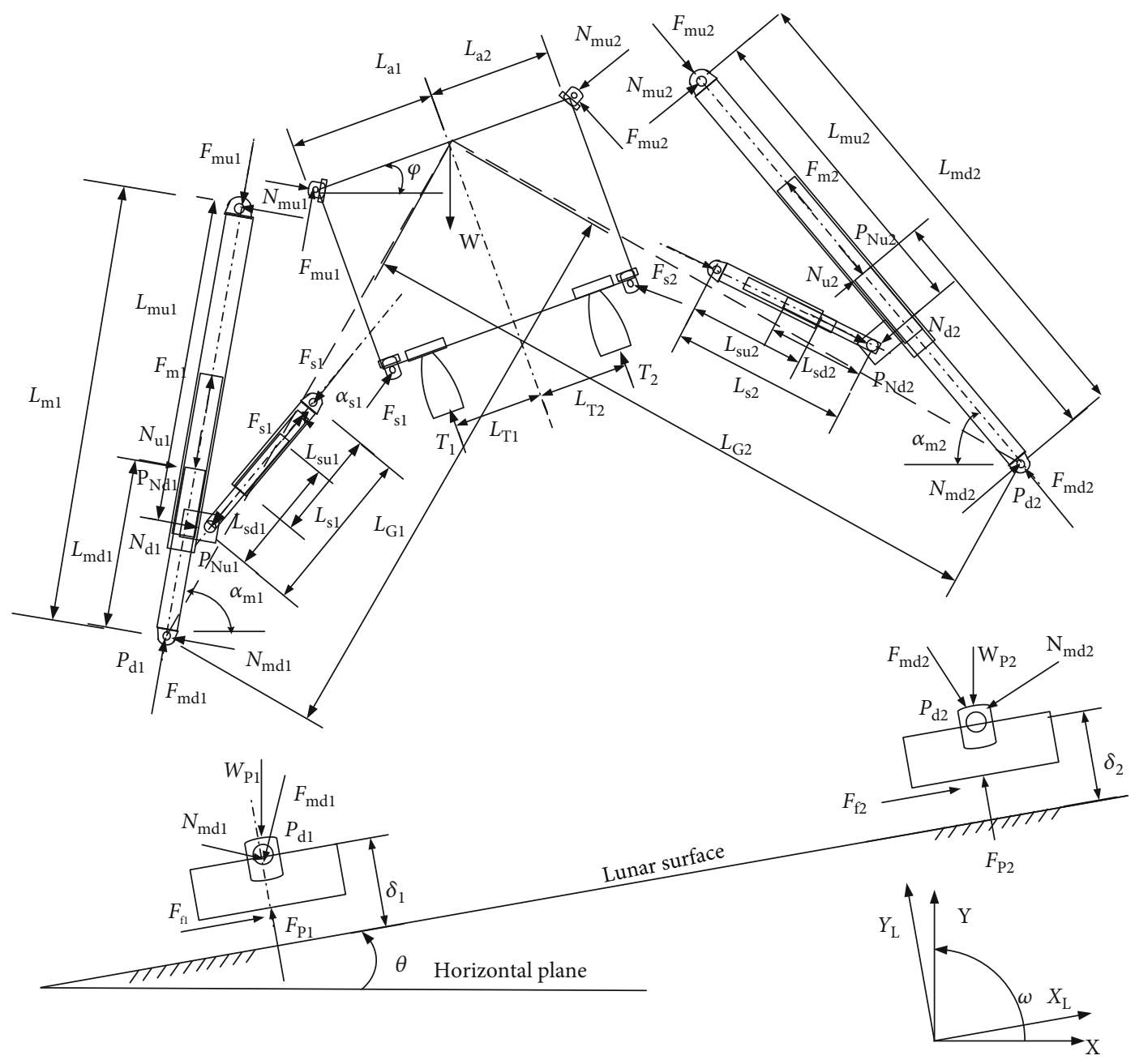

FIgURE 1: Lunar lander 2-2 mode soft-landing force analysis.

As per the quantitative moment of momentum relative to the center of mass, the equation for the rotation of the body through the center of mass around the $O Z$ rotating coordinate system is as follows:

$$
\begin{aligned}
I \ddot{\varphi}= & -F_{m u 1} \sin \left(\alpha_{m 1}-\varphi\right) \cdot L_{a 1}+F_{m u 2} \sin \left(\alpha_{m 2}+\varphi\right) \cdot L_{a 2}+N_{m u 1} \cos \left(\alpha_{m 1}-\varphi\right) \\
& \cdot L_{a 1}-N_{m u 2} \cos \left(\alpha_{m 2}+\varphi\right) \cdot L_{a 2}-F_{s 1} \sin \left(\alpha_{s 1}-\varphi\right) \cdot L_{a 1}+F_{s 1} \cos \left(\alpha_{s 1}-\varphi\right) \\
& \cdot L_{b 1}+F_{s 2} \sin \left(\alpha_{s 2}+\varphi\right) \cdot L_{a 2}-F_{s 2} \cos \left(\alpha_{s 2}+\varphi\right) \cdot L_{b 2}-T_{1} \cdot L_{T 1}+T_{2} \cdot L_{T 2}
\end{aligned}
$$

In Equations (1)-(3), $x$ and $y$ are body part coordinates at the $O X$ and $O Y$ axes in the inertial coordinate system. $F_{s u 1}$ and $F_{s u 2}$ are the respective axial forces of the two secondary struts on the hinge point of the body. $I$ is the moment of inertia of the body. $L_{a 1}$ and $L_{a 2}$ are the respective distances from the two hinge points of the body to the geometric centerline of the body. $F_{s 1}$ and $F_{s 2}$ are the respective forces applied on the body by the two secondary struts.

The mass of the landing buffer struts and foot pad part on the left-hand side in Figure 1 is $m_{p 1}$, and the dynamic equation in the vertical direction of the inertial coordinate system $O-X Y$ is as follows:

$m_{p 1} \ddot{y}_{p 1}=F_{p 1} \cos \theta+F_{f 1} \sin \theta-F_{m d 1} \sin \alpha_{m 1}-N_{m d 1} \cos \alpha_{m 1}-W_{p 1}$.

The dynamic equation in the horizontal direction is as follows:

$$
m_{p 1} \ddot{x}_{p 1}=-F_{p 1} \sin \theta+F_{f 1} \cos \theta-F_{m d 1} \cos \alpha_{m 1}+N_{m d 1} \sin \alpha_{m 1} .
$$


TABLE 1: Notifications of landing force of lunar lander under 2-2 mode.

\begin{tabular}{|c|c|}
\hline Symbol & Definition \\
\hline$F_{m i}$ & Internal force of the primary buffer strut \\
\hline$F_{m d i}$ & Axial force from the primary strut to foot pad hinge joint \\
\hline$F_{m u i}$ & Axial force from the primary strut to body hinge joint \\
\hline$F_{p i}$ & Vertical force from lunar surface to foot pad \\
\hline$F_{f i}$ & Force along the lunar surface from lunar surface to foot pad \\
\hline$L_{b i}$ & Height of lander framework \\
\hline$L_{G i}$ & Distance from body gravity center to foot pad $i$ \\
\hline$L_{m i}$ & Primary buffer strut length \\
\hline$L_{m d i}$ & Lower strut length of primary strut \\
\hline$L_{m u i}$ & Upper sleeve length on the primary buffer strut \\
\hline$L_{s i}$ & Secondary buffer strut length \\
\hline$L_{s i 0}$ & Secondary buffer strut length in the stretching status \\
\hline$L_{s d i}$ & Lower strut length of secondary strut \\
\hline$L_{\text {sui }}$ & Upper sleeve length on the secondary buffer strut \\
\hline$L_{T i}$ & Distance between trimming rocket engine and body gravity center \\
\hline$m$ & Elastic mass \\
\hline$m_{p i}$ & Inelastic mass \\
\hline$N_{m d i}$ & Vertical force from the primary buffer strut to foot pad hinge joint \\
\hline$N_{m u i}$ & Vertical force from the primary buffer strut to body hinge joint \\
\hline$P_{d i}$ & Hinge joint between primary strut and foot pad \\
\hline$P_{N d i}$ & Hinge joint between primary strut and secondary strut \\
\hline$P_{\mathrm{Nui}}$ & Touch point between upper sleeve of primary strut and end of the lower strut \\
\hline$P_{u i}$ & Hinge joint between primary strut and body \\
\hline$T_{i}$ & Force on the lander by trimming rocket engine \\
\hline$W$ & Gravity of lander body \\
\hline$W_{P i}$ & Gravity of lander foot pad \\
\hline$\theta$ & Lunar surface angle, counterclockwise is positive \\
\hline$\varphi$ & Body gesture angle, counterclockwise is positive \\
\hline$\omega$ & Angle velocity of body gesture, counterclockwise is positive \\
\hline$\delta_{i}$ & Sinking depth of foot pad \\
\hline$\beta_{s i}$ & Angle between two secondary struts \\
\hline$a_{m i}$ & Angle between primary strut and horizontal plane, counterclockwise is positive \\
\hline$a_{s i}$ & Angle between secondary strut and horizontal plane, counterclockwise is positive \\
\hline Index 0 & Represents initial value \\
\hline Index $i=1,2$ & Represents the landing legs or trimming rockets 1 and 2 , respectively \\
\hline
\end{tabular}

TABLE 2: Work condition of soft-landing simulation analysis.

\begin{tabular}{lccccccc}
\hline $\begin{array}{l}\text { Throwing } \\
\text { mass }(\mathrm{kg})\end{array}$ & $\begin{array}{c}\text { Landing } \\
\text { mode }\end{array}$ & $\begin{array}{c}\text { Vertical velocity } \\
(\mathrm{m} / \mathrm{s})\end{array}$ & $\begin{array}{c}\text { Inclination angle of lunar } \\
\text { surface }\left(^{\circ}\right)\end{array}$ & $\begin{array}{c}\text { Initial pitch } \\
\text { angle }\left({ }^{\circ}\right)\end{array}$ & $\begin{array}{c}\text { Landing leg } \\
\text { touching order }\end{array}$ & $\begin{array}{c}\text { Lunar soil } \\
\text { state }\end{array}$ & $\begin{array}{c}\text { The gravity } \\
\text { field }\end{array}$ \\
\hline 1350 & $2-2$ & 4.0 & 9 & 0 & $2 / 3-1 / 4$ & Flexible & The moon \\
\hline
\end{tabular}




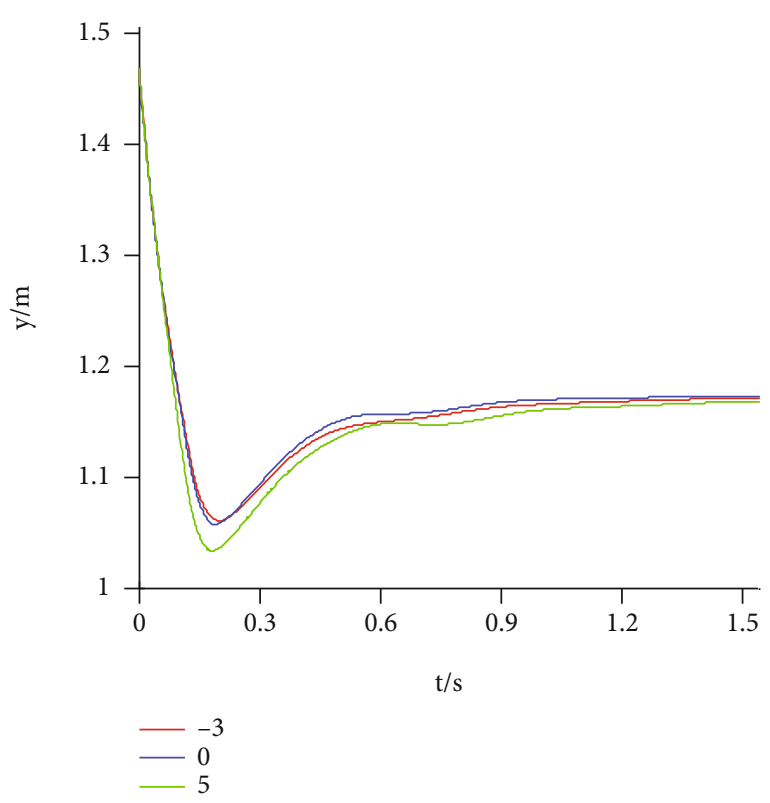

FIgURE 2: Body displacement versus time curve.

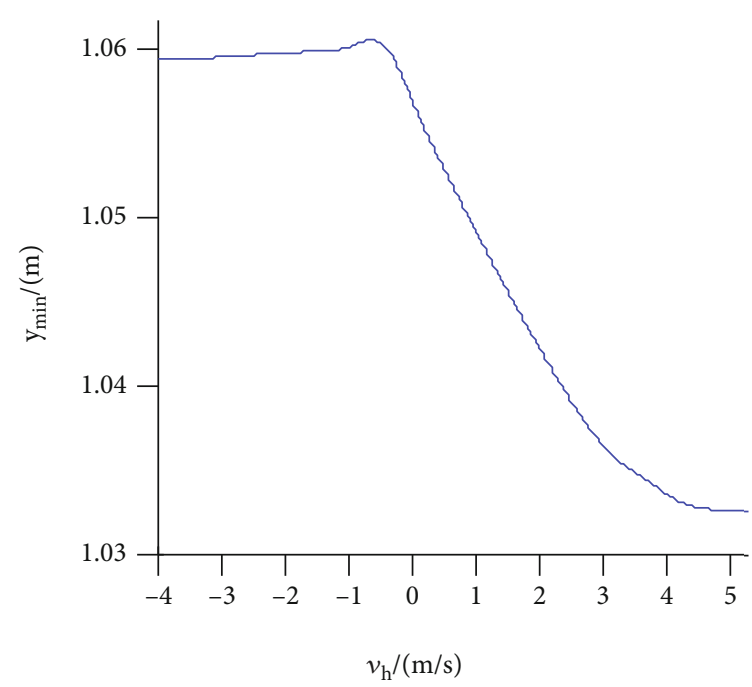

FIGURE 3: Minimum displacement curve of the body from the lunar surface.

The mass of the landing strut and foot pad on the righthand side is $m_{p 2}$. In the inertial coordinate system $O-X Y$, the dynamic equation in the vertical direction is as follows:

$m_{p 2} \ddot{y}_{p 2}=F_{p 2} \cos \theta+F_{f 1} \sin \theta-F_{m d 2} \sin \alpha_{m 2}+N_{m d 2} \cos \alpha_{m 2}-W_{p 2}$.

The dynamic equation in the horizontal direction is as follows:

$m_{p 2} \ddot{x}_{p 2}=-F_{p 2} \sin \theta+F_{f 2} \cos \theta+F_{m d 2} \cos \alpha_{m 2}+N_{m d 2} \sin \alpha_{m 2}$.

\section{Impact on Soft-Landing Performance by Initial Horizontal Landing Velocity}

The vertical velocity of the lunar lander during landing could make shock load in the vertical direction at the moment of landing. When the vertical velocity is too large, it may cause the buffer to exceed the maximum buffer stroke, and make the overall damage of the lander. On the other hand, if the horizontal velocity is too big, the force against the buffer will be severely unbalanced, especially on the secondary buffer where the tension and pressure will no longer be synchronized. This unbalanced force will lead to a rollover and instability of the lander. Especially on the lunar slope surface, when the horizontal landing velocity is too big, it is more likely to cause the spacecraft instability. When landing down the lunar surface, the lander may be dumped and overturned due to excessive horizontal landing velocity. When the lander lands on the lunar surface along uphill, the horizontal landing velocity is too large, which may cause the impact force of the primary/secondary strut to be too large, resulting in damage and instability of the primary/secondary strut. Therefore, the initial horizontal velocity of the lunar lander has a certain impact on the soft-landing performance. Especially the landing along the lunar slope, the initial horizontal velocity has an important impact on the soft-landing buffer characteristics and stability. The impact of initial horizontal velocity on the soft-landing performance of the lunar lander is extremely necessary and critical. Table 2 shows the work condition of soft-landing simulation analysis.

3.1. Impact on the Body Displacement by Initial Horizontal Velocity. Figure 2 is a time domain diagram of the displacement of the lunar lander body at different initial horizontal velocities. Figure 3 shows the relationship between the minimum displacement of the body from the lunar surface and the initial horizontal velocity. When $v_{\mathrm{h}}<0$, it indicates that the lander lands uphill along the lunar surface. When $v_{\mathrm{h}}>$ 0 , it indicates that the lander lands downhill along the lunar surface. It can be seen from Figure 2 that the time domain of the displacement of the lunar lander is basically the same no matter it vertically lands on the lunar surface slope without horizontal velocity, or lands along the uphill slope, or lands along the downhill slope. As shown in Figure 3, when the initial horizontal velocity $v_{\mathrm{h}} \in[-4,4]$, the maximum and minimum values of the minimum distance from the lunar surface are 1.061 meters and 1.032 meters, respectively. The difference is very small. Table 3 compares the maximum/minimum initial level $\left(v_{\mathrm{h}}= \pm 4 \mathrm{~m} / \mathrm{s}\right)$ along the upper (lower) slope and the minimum distance from the lunar surface in free fall $\left(v_{\mathrm{h}}=0 \mathrm{~m} / \mathrm{s}\right)$. The error of $v_{\mathrm{h}}=-4 \mathrm{~m} / \mathrm{s}$ with $v_{\mathrm{h}}=0$ is $0.28 \%$, and the error between $v_{\mathrm{h}}=4 \mathrm{~m} / \mathrm{s}$ and $v_{\mathrm{h}}=$ 0 is $-2.36 \%$. It can be seen that the initial horizontal velocity has little impact on the minimum distance of the body from the lunar surface, no matter the lunar lander is landing along the lunar surface uphill or downhill.

3.2. Impact on Body Overload by Initial Horizontal Velocity. Figure 4 is a time domain diagram of the body's overload at different initial horizontal velocities of the lunar lander. It 
TABLE 3: Impact of typical working conditions of initial horizontal velocity on the minimum displacement of the body.

\begin{tabular}{lccc}
\hline Landing conditions & Initial horizontal velocity $(\mathrm{m} / \mathrm{s})$ & Minimum displacement of the body $(\mathrm{m})$ & Error* \\
\hline Free fall & 0 & 1.057 & $/$ \\
Landing uphill & -4 & 1.06 & $0.28 \%$ \\
Landing downhill & 4 & 1.032 & $-2.36 \%$
\end{tabular}

(1) Error $=($ result of initial horizontal velocity - result of free fall $) /$ result of free fall

(2) The errors in the following tables are calculated according to this one

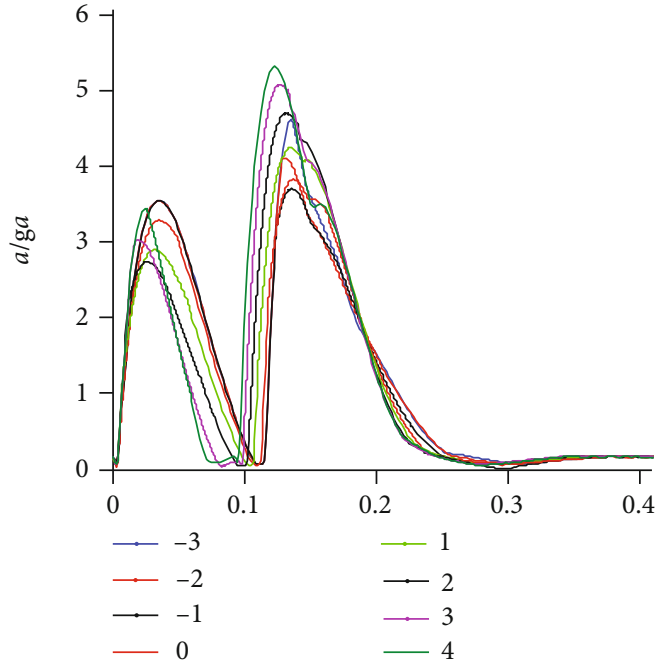

Figure 4: Body overload curve vs. time.

can be seen from the figure that there are two peaks of the overload of the lunar lander body. This is because the flexible body, with its natural mode, produces a physical phenomenon of a wide dynamic response to the exciting force exerted by a single set of landing buffer mechanisms. The two peaks are the dynamic responses of the respective groups of shock forces when the landing leg touches the ground. And the second peak is always greater than first peak which appears at about 0.05 seconds, and the value is about $3.0 g_{n} \sim 3.6 g_{n}\left(g_{n}\right.$ is the Earth's gravity acceleration). The second peak appears around 0.15 seconds where the value is about $3.8 g_{n} \sim 5.4 g_{n}$. After 0.25 seconds, the body overload decays to zero slowly.

Figure 5 shows the relationship between the maximum overload of the body of lunar lander and the initial horizontal velocity. As can be seen from the figure, when the lunar lander lands uphill along the lunar surface as the initial horizontal velocity is $3 \mathrm{~m} / \mathrm{s} \sim 4 \mathrm{~m} / \mathrm{s}$, the maximum overload of the body is $4.6 g_{n}$. When the initial horizontal velocity is less than $3 \mathrm{~m} / \mathrm{s}$, the maximum overload of the lander body decreases with the decrease of the initial horizontal velocity. When the initial horizontal velocity is $0.8 \mathrm{~m} / \mathrm{s}$, the minimum value of the maximum overload of the lander body is $3.6 g_{n}$. When the lunar lander landed downhill along the lunar surface, the maximum overload of the lander body is increasing with the initial horizontal velocity. When the initial horizon-

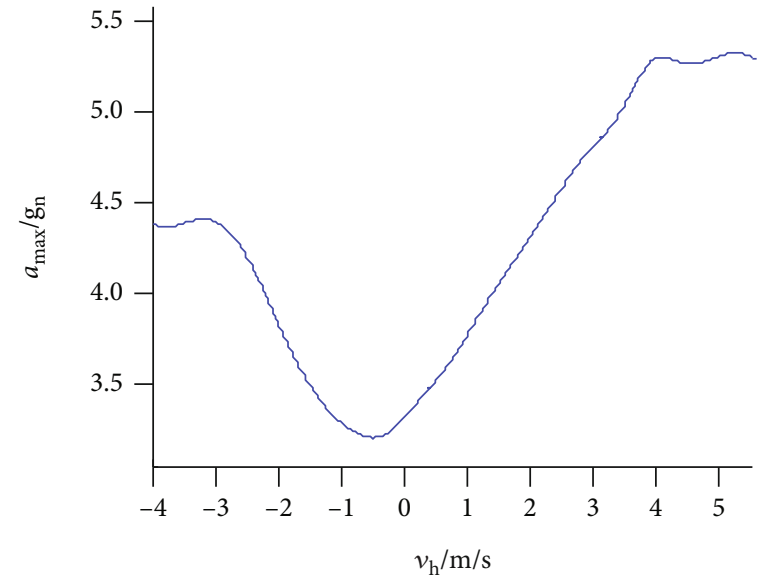

Figure 5: Maximum overload vs. initial horizontal velocity.

TABLE 4: Impact of initial horizontal velocity landing conditions on the maximum overload of the body.

\begin{tabular}{lccc}
\hline $\begin{array}{l}\text { Landing } \\
\text { condition }\end{array}$ & $\begin{array}{c}\text { Initial horizontal velocity } \\
(\mathrm{m} / \mathrm{s})\end{array}$ & $\begin{array}{c}\text { Body } \\
\text { overload }\end{array}$ & Error \\
\hline Free fall & 0 & $3.8 g_{n}$ & $/$ \\
Landing uphill & -4 & $4.6 g_{n}$ & $21 \%$ \\
Landing downhill & 4 & $5.2 g_{n}$ & $36.8 \%$ \\
\hline
\end{tabular}

tal velocity reaches $3.5 \mathrm{~m} / \mathrm{s}$, the maximum overload of the lander body is $5.2 \mathrm{~g}_{n}$. When the initial horizontal velocity is greater than $3.5 \mathrm{~m} / \mathrm{s}$, the maximum overload of the lander body is basically a constant value which is $5.2 g_{n}$.

Table 4 compares the maximum overload of the body at the maximum and minimum initial levels $\left(v_{\mathrm{h}}= \pm 4 \mathrm{~m} / \mathrm{s}\right)$ and free fall $\left(v_{\mathrm{h}}=0 \mathrm{~m} / \mathrm{s}\right)$ along the uphill (downhill) slope. The error is $21 \%$ when $v_{\mathrm{h}}=-4 \mathrm{~m} / \mathrm{s}$ and $v_{\mathrm{h}}=0$ and $36.8 \%$ when $v_{\mathrm{h}}=4 \mathrm{~m} / \mathrm{s}$ and $v_{\mathrm{h}}=0$. It can be seen that the initial horizontal velocity has a certain impact on the overload of the body when the lunar lander lands on the slope of the moon uphill or downhill.

3.3. Impact on the Primary Strut by Initial Horizontal Velocity. Figure 6 shows the relationship between the maximum force of the primary strut 1 and the initial horizontal velocity. It can be seen that when the lander is landing along the lunar 


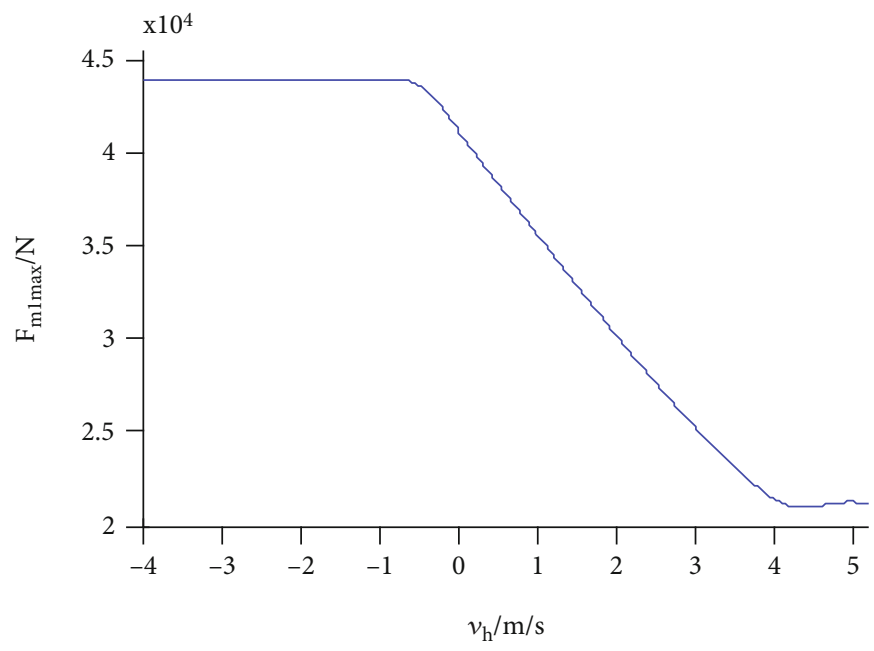

FIgURE 6: The maximum force on the primary strut 1 vs. the initial horizontal velocity.

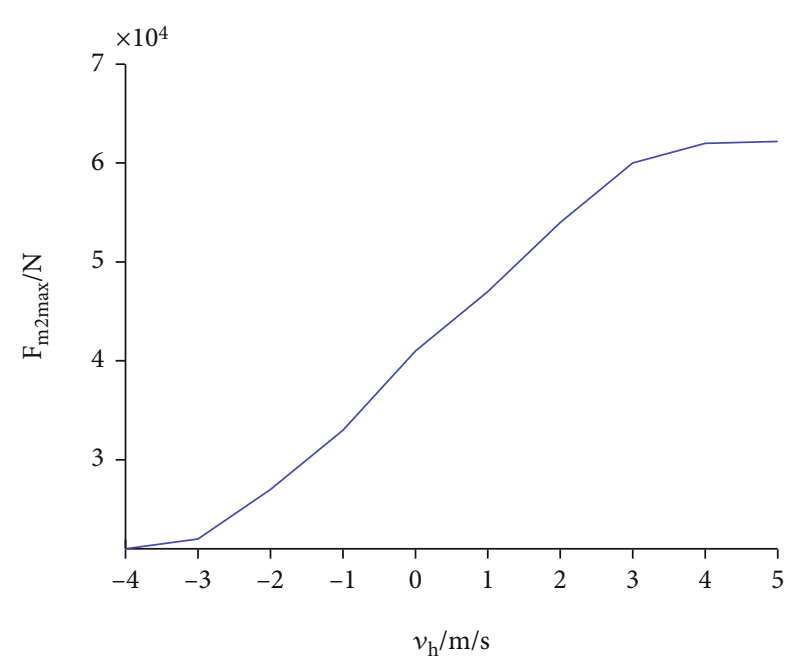

FIGURE 7: The maximum force on the primary strut 2 vs. the initial horizontal velocity.

surface uphill, the maximum force on the primary strut 1 is constant, which is $4.3 \times 10^{4} \mathrm{~N}$. When the lander lands downhill along the lunar surface, the maximum force on the primary strut 1 decreases with the increase of the initial horizontal landing velocity. When the initial horizontal landing velocity is $4 \mathrm{~m} / \mathrm{s}$, the maximum force of the primary strut 1 decays to $2.1 \times 10^{4} \mathrm{~N}$.

Figure 7 shows the relationship between the maximum force of the primary strut 2 and the initial horizontal velocity. It can be seen from the figure that when the lander is landing along the lunar surface uphill, the initial horizontal velocity is decremented with the maximum force of the primary strut 2 . The greater the initial horizontal velocity is, the smaller the maximum force on primary strut 2 is. When the initial horizontal velocity is $4 \mathrm{~m} / \mathrm{s}$, the primary strut 2 has a maximum force of $2.1 \times 10^{4} \mathrm{~N}$. When the lander lands downhill along the lunar surface, the maximum force on the primary strut 2 increases as the initial horizontal velocity is increasing. When the initial horizontal velocity is $0 \sim 3 \mathrm{~m} / \mathrm{s}$, the maximum force change on the primary strut 2 is relatively severe. When the initial horizontal velocity is in the range from 3 to $5 \mathrm{~m} / \mathrm{s}$, the maximum force change on the primary strut 2 is relatively slow.

Table 5 compares the maximum force on the primary strut at the maximum and minimum initial levels $\left(v_{\mathrm{h}}= \pm 4 \mathrm{~m} / \mathrm{s}\right)$ and the free fall $\left(v_{\mathrm{h}}=0 \mathrm{~m} / \mathrm{s}\right)$ in the uphill (downhill) slope landing. When $v_{\mathrm{h}}=-4 \mathrm{~m} / \mathrm{s}$, the maximum force on the primary strut $1 / 2$ is $4.3 \times 10^{4} \mathrm{~N} / 2.0 \times 10^{4} \mathrm{~N}$, respectively, and the errors with $v_{\mathrm{h}}=0$ is $4.88 \% /-50 \%$, respectively; When $v_{\mathrm{h}}=4 \mathrm{~m} / \mathrm{s}$, the primary strut $1 / 2$ of the maximum force is $2.1 \times 10^{4} \mathrm{~N} / 6.1 \times 10^{4} \mathrm{~N}$, respectively, and the errors of $v_{\mathrm{h}}=0$ are $-48.78 \% / 52.5 \%$, respectively. It can be seen that when the lunar lander lands uphill along the lunar surface, the initial horizontal velocity has less impact on the maximum force on the primary strut 1 and has a greater impact on the maximum force on the primary strut 2. When landing along the downhill slope, the initial horizontal velocity has a great impact on both primary struts $1 / 2$; meanwhile, the force on the primary strut 1 significantly decreases. In the case where the initial horizontal velocity is the same, the peak force of the primary strut 1 when landing downhill is much larger than that along the uphill slope.

3.4. Impact on Secondary Strut by Initial Horizontal Velocity. Figure 8 shows the relationship between the maximum force on the secondary strut 1 and the initial horizontal velocity. It can be seen from the figure that when the lander lands on the uphill slope, the initial horizontal velocity has no impact on the maximum force on the secondary strut 1 . The maximum force of the secondary strut 1 is a constant value of $0.75 \times 10^{4} \mathrm{~N}$. When the lander lands downhill, the maximum force on the secondary strut 1 increases when the initial horizontal velocity increases. When the horizontal velocity reaches $4 \mathrm{~m} / \mathrm{s}$, the maximum force of the secondary strut 1 reaches its max value of $6.2 \times 10^{4} \mathrm{~N}$.

Figure 9 shows the relationship between the maximum force of the secondary strut 2 and the initial horizontal 
TABLE 5: Impact of initial horizontal velocity on the maximum force on primary strut.

\begin{tabular}{lccc}
\hline Landing condition & Initial horizontal velocity $(\mathrm{m} / \mathrm{s})$ & Max force on primary strut $1 / 2(\mathrm{~N})$ & Error \\
\hline Free fall & 0 & $4.1 \times 10^{4} / 4.0 \times 10^{4}$ & $/$ \\
Landing uphill & -4 & $4.3 \times 10^{4} / 2.0 \times 10^{4}$ & $4.88 \% /-50 \%$ \\
Landing downhill & 4 & $2.1 \times 10^{4} / 6.1 \times 10^{4}$ & $-48.78 \% / 52.5 \%$ \\
\hline
\end{tabular}

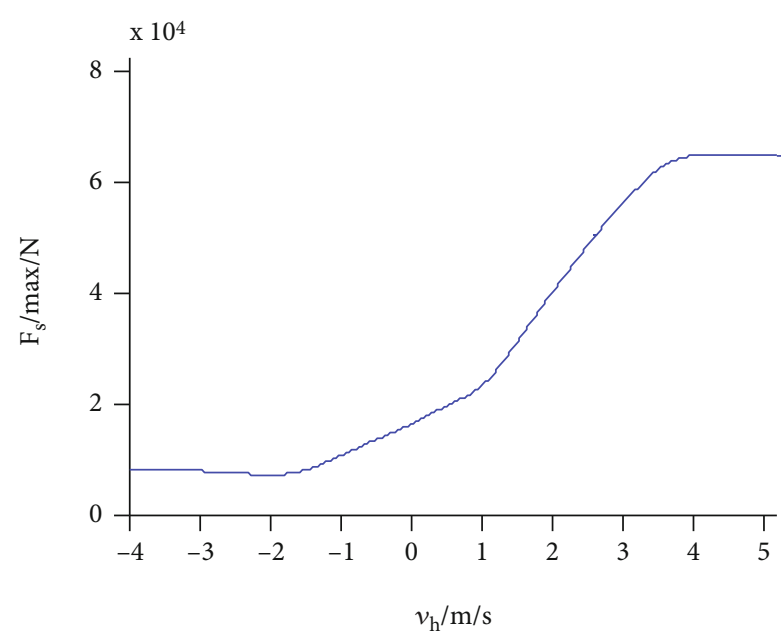

Figure 8: The maximum force of the secondary strut 1 vs. the initial horizontal velocity.

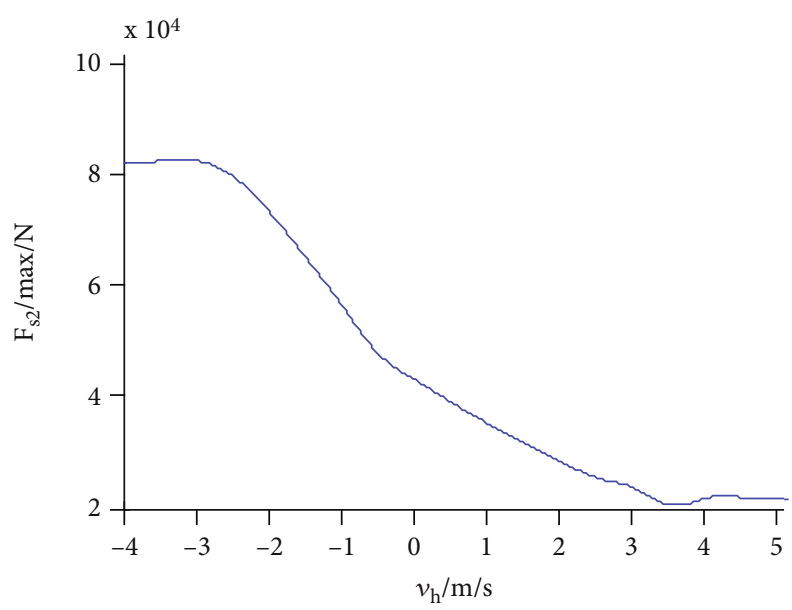

Figure 9: The maximum force of the secondary strut 2 vs. the initial horizontal velocity.

velocity. It can be seen from the figure that when the initial horizontal velocity of the lander along the uphill reaches $3 \mathrm{~m} / \mathrm{s}$, the maximum force of the secondary strut 2 reaches the maximum value of $8.1 \times 10^{4} \mathrm{~N}$. When the initial horizontal velocity of the lander downhill reaches $3.5 \mathrm{~m} / \mathrm{s}$, the maximum force of the secondary strut 2 reaches a minimum of $2.1 \times 10^{4} \mathrm{~N}$.
Table 6 compares the maximum force on the secondary strut at the maximum and minimum initial levels $\left(v_{\mathrm{h}}= \pm 4 \mathrm{~m} / \mathrm{s}\right)$ and the free fall $\left(v_{\mathrm{h}}=0 \mathrm{~m} / \mathrm{s}\right)$ in the uphill (downhill) slope landing. When $v_{\mathrm{h}}=-4 \mathrm{~m} / \mathrm{s}$, the maximum force on the secondary strut $1 / 2$ is $0.75 \times 10^{4} \mathrm{~N} / 8.1 \times 10^{4} \mathrm{~N}$ , respectively, and the error with $v_{\mathrm{h}}=0$ is $-50 \% / 92.8 \%$, respectively. When $v_{\mathrm{h}}=4 \mathrm{~m} / \mathrm{s}$, the maximum force on the secondary strut $1 / 2$ is $6.2 \times 10^{4} \mathrm{~N} / 2.2 \times 10^{4} \mathrm{~N}$, respectively, and the error with $v_{\mathrm{h}}=0$ is $313.3 \% /-47.6 \%$, respectively. It can be seen that the initial horizontal velocity has a great impact on the maximum force on the secondary strut $1 / 2$ no matter its landing along the downhill or uphill slope. Meanwhile, if the initial horizontal velocities are the same, the force in landing along downhill slope is much greater than landing along the uphill. The force on secondary strut 2 is much greater when landing along the uphill slope than on the downhill slope.

According to the analysis above, the impact of the initial horizontal velocity on the soft-landing performance can be concluded as follows:

(1) The initial horizontal velocity has no impact on the minimum distance from the moon

(2) The initial horizontal velocity has a certain impact on the overload of the body, which will increase the overload of the body

(3) The initial horizontal velocity has a great impact on the maximum force of the primary strut 2 . The impact on the primary strut 1 is big when landing along the downhill slope and small when landing along the lunar slope

(4) The initial horizontal velocity has a great impact on the maximum force on secondary strut both $1 / 2$. Especially when landing along the uphill slope, structural damage or instability on the secondary strut 2 could occur due to the excessive force on it

\section{Impact on Soft-Landing Performance by Initial Pitch Angle}

The angle between the body and the horizontal plane during the landing on the moon is the pitch angle. Usually, the pitch angle of the lunar lander is difficult to adjust to the ideal state by the control system due to various factors. Especially during the process of landing, it is difficult to make the body completely horizontal. Therefore, the initial pitch angle of the lunar lander has critical impact on soft-landing 
TABLE 6: Impact of initial horizontal velocity on the maximum force on secondary strut.

\begin{tabular}{lccc}
\hline Landing conditions & Initial horizontal velocity $(\mathrm{m} / \mathrm{s})$ & Maximum force on secondary strut $1 / 2(\mathrm{~N})$ & Error \\
\hline Free fall & 0 & $1.5 \times 10^{4 /} 4.2 \times 10^{4}$ & $/$ \\
Landing uphill & -4 & $0.75 \times 10^{4 /} 8.1 \times 10^{4}$ & $-50 \% / 92.8 \%$ \\
Landing downhill & 4 & $6.2 \times 10^{4 / 2} 2.2 \times 10^{4}$ & $313.3 \% /-47.6 \%$ \\
\hline
\end{tabular}

TABLE 7: Lunar lander soft-landing simulation analysis.

\begin{tabular}{lccccccc}
\hline $\begin{array}{l}\text { Throwing } \\
\text { mass }(\mathrm{kg})\end{array}$ & $\begin{array}{c}\text { Landing } \\
\text { mode }\end{array}$ & $\begin{array}{c}\text { Vertical } \\
\text { velocity }(\mathrm{m} / \mathrm{s})\end{array}$ & $\begin{array}{c}\text { Horizontal } \\
\text { velocity }(\mathrm{m} / \mathrm{s})\end{array}$ & $\begin{array}{c}\text { Inclination angle of lunar } \\
\text { surface }\left(^{\circ}\right)\end{array}$ & $\begin{array}{c}\text { Landing leg } \\
\text { touching order }\end{array}$ & $\begin{array}{c}\text { Lunar soil } \\
\text { state }\end{array}$ & $\begin{array}{c}\text { The gravity } \\
\text { field }\end{array}$ \\
\hline 1350 & $2-2$ & 4.0 & 0 & 0 & $2 / 3-1 / 4$ & Flexible & The moon \\
\hline
\end{tabular}

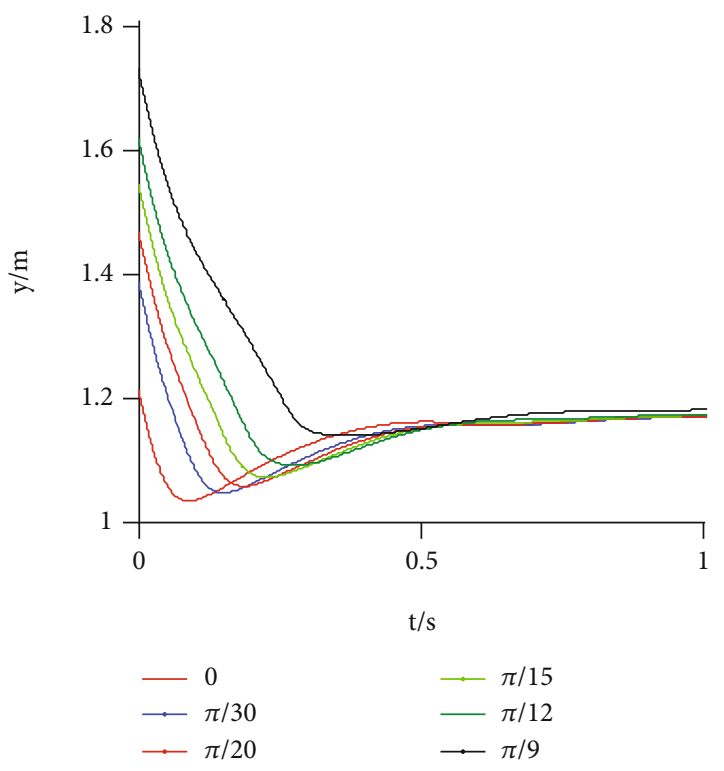

Figure 10: Body displacement vs. time.

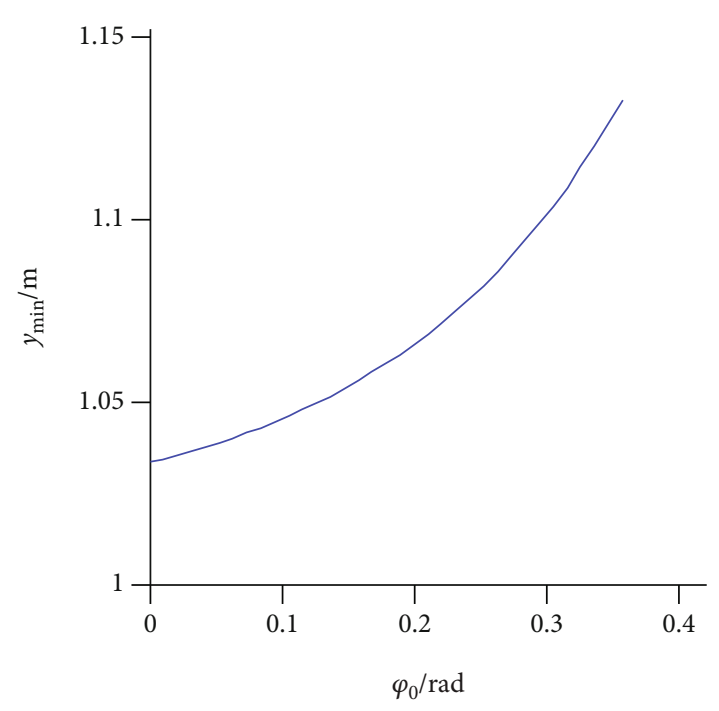

FIGURE 11: Minimum displacement of the body from the lunar surface. performance which is extremely necessary and important. Table 7 shows the simulation analysis of the lunar lander soft landing.

4.1. Impact on Body Displacement by Initial Pitch Angle. Figure 10 is a time domain diagram of the lunar lander displacement at different initial pitch angles. It can be seen from the figure that as the initial pitch angle of the body increases, the displacement of the body changes significantly with time going forward. Meanwhile, the displacement of the body from the lunar surface is getting longer. When the initial pitch angle of the body is $0^{\circ}$, the displacement of the body from the lunar surface shows minimum value at 0.1 seconds. When the initial pitch angle of the body is $\pi / 9$ , the displacement of the body from the lunar surface reaches minimum value at 0.35 seconds.

Figure 11 is a graph showing the relationship between the minimum displacement of the body from the lunar surface and the initial pitch angle. It can be seen from the figure that as the initial pitch angle of the body increases, the minimum displacement of the body from the lunar surface increases. When the initial pitch angle of the body is $0^{\circ}$, the minimum value of the minimum displacement from the lunar surface is 1.04 meters. When the initial pitch angle of the body is $\pi / 9$, the minimum displacement of the body from the lunar surface is 1.14 meters.

Table 8 compares the minimum displacement from the lunar surface when the initial pitch angle is $0^{\circ}$ as well as its maximum degree $20^{\circ}$. The error between them is $9.6 \%$. It can be seen that the initial pitch angle has small impact on the minimum displacement of the body from the lunar surface.

4.2. Impact on Body Overload by Initial Pitch Angle. Figure 12 is a time domain diagram of the lunar lander overload at different initial pitch angles. It can be seen from the figure that when the initial pitch angle of the body is 0 , there is only one peak. When the initial pitch angle of the body is $0 \sim \pi / 15$, there are two peaks where the second peak is greater than the first one. The time interval between the two peaks increases when the pitch angle increases. When the initial pitch angle of the body is greater than $\pi / 15$, the body 
TABLE 8: Impact of typical working conditions of initial pitch angle on the minimum displacement of the body.

\begin{tabular}{lccr}
\hline Initial pitch angle & Minimum displacement of the body $(\mathrm{m})$ & Error $*$ & Note \\
\hline $0^{\circ}$ & 1.04 & $/$ & Minimum \\
$20^{\circ}(\pi / 9)$ & 1.14 & $9.6 \%$ & Maximum
\end{tabular}

(1) Error $=\left(\right.$ result of pitch angle - result of initial pitch angle $\left.0^{\circ}\right) /$ initial pitch angle of $0^{\circ}$

(2) The errors in the following tables are calculated according to this one

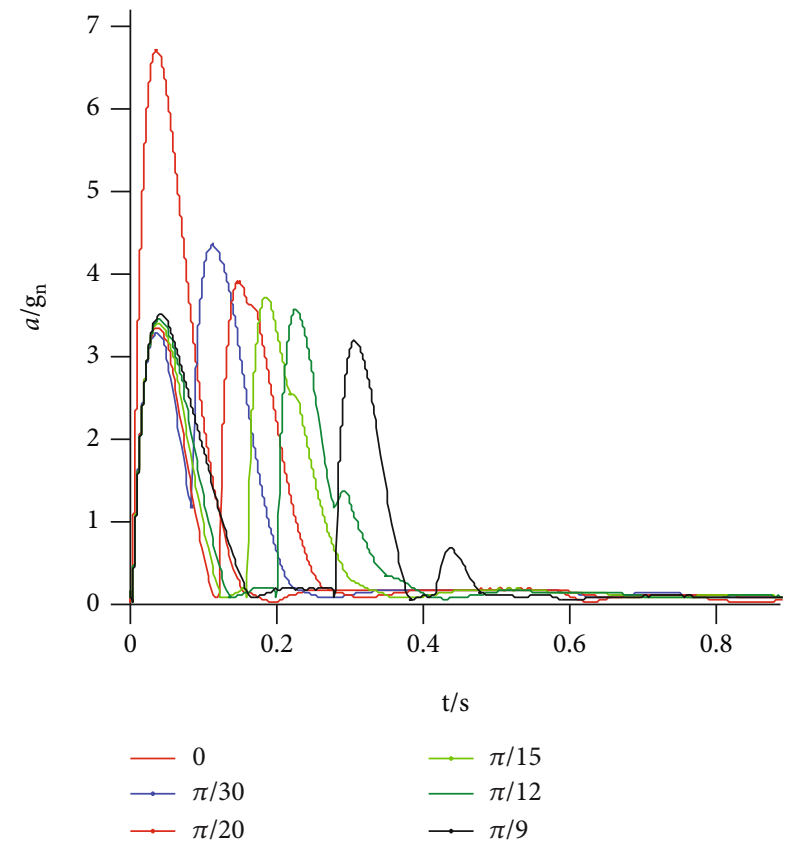

FIgURE 12: Body overload vs. time.

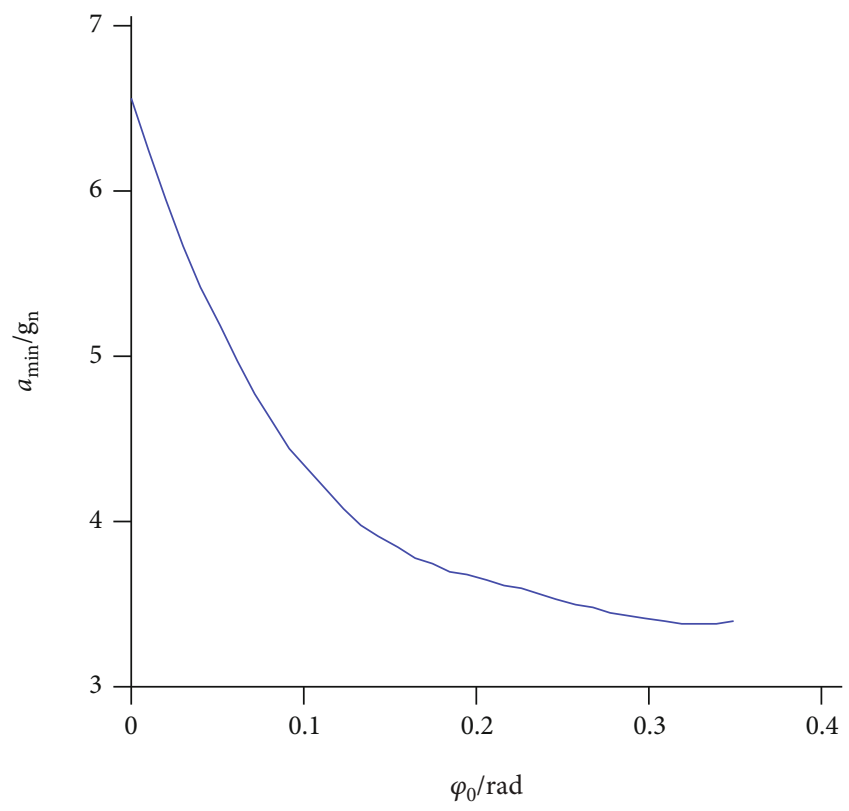

FIgURE 13: Maximum of body overload vs. initial pitch angle.
TABLE 9: Impact of typical working conditions of initial pitch angle on maximum overload of the body.

\begin{tabular}{lccc}
\hline Initial pitch angle & Maximum body overload & Error & Note \\
\hline $0^{\circ}$ & $6.5 g_{n}$ & $/$ & Maximum \\
$20^{\circ}(\pi / 9)$ & $3.3 g_{n}$ & $-49.2 \%$ & Minimum \\
\hline
\end{tabular}

overload has three peaks where the time intervals between the peaks increase when the initial pitch angle increases.

Figure 13 is a graph showing the relationship between the maximum overload of the lunar lander and the initial pitch angle. It can be seen from the figure that the maximum overload of the lunar lander is decreasing when the initial pitch angle of the body increases. When the initial pitch angle is 0 , the maximum overload of the body is $6.5 g_{n}$; when the initial pitch angle is $\pi / 9$, the maximum overload of the body is $3.3 g_{n}$.

Table 9 compares the maximum overload of the body when the initial pitch angle is $0^{\circ}$ and $20^{\circ}$ which is the maximum degree. The error between them is $-49.2 \%$. It can be seen that the initial pitch angle has a greater impact on the overload of the body.

4.3. Impact on the Primary Strut by Initial Pitch Angle. Figure 14 shows the relationship between the maximum force of the primary strut 1 and the initial pitch angle. It can be seen from the figure that the maximum force of the primary strut 1 increases when the initial pitch angle increases. When the initial pitch angle is 0 , the force on the primary strut 1 is $3.7 \times$ $10^{4} \mathrm{~N}$. When the initial pitch angle is $\pi / 9$, the maximum force on the primary strut 1 is $4.22 \times 10^{4} \mathrm{~N}$. It can be seen that the initial pitch angle of the body has little impact on the maximum force on the primary strut 1.

Figure 15 is a graph showing the relationship between the maximum force of the primary strut 2 and the initial pitch angle. It can be seen from the figure that when the initial pitch angle of the body is from 0 to $0.1 \mathrm{rad}$, the maximum force of the primary strut 2 increases with the increase of the initial pitch angle. When the initial pitch angle is $0.1 \mathrm{rad}$, the maximum force of the primary strut 2 is $4.1 \times 10^{4} \mathrm{~N}$. When the initial pitch angle of the body is greater than $0.1 \mathrm{rad}$, the maximum force of the primary strut 2 decreases with the increase of the initial pitch angle. When the initial pitch angle is $0.35 \mathrm{rad}$, the maximum force of the primary strut 2 is $3.6 \times 10^{4} \mathrm{~N}$. It can be seen that the initial pitch angle of the body does not show significant impact on the maximum force of the primary strut 2 . 


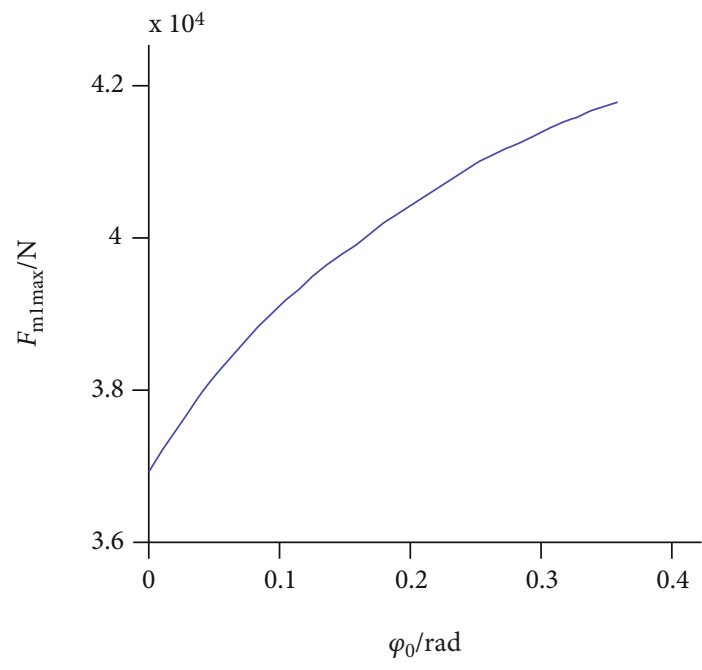

FIGURE 14: Max force on the primary strut 1 vs. initial pitch angle.

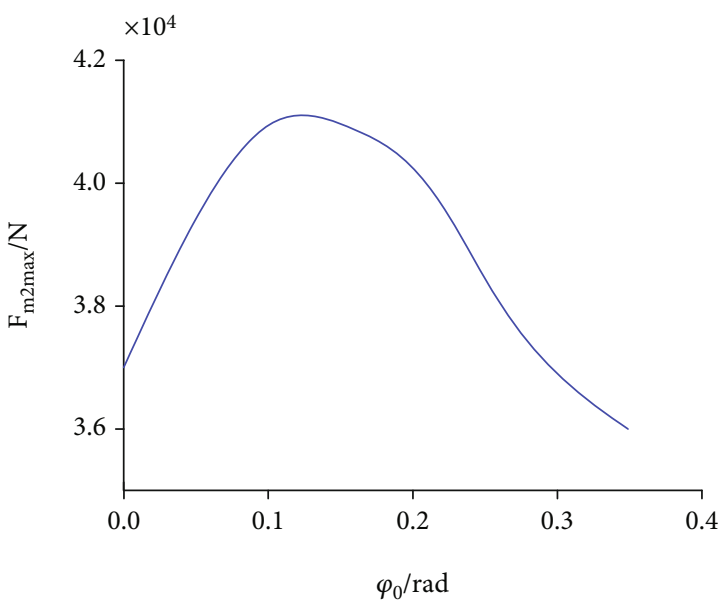

FIGURE 15: Max force on the primary strut 2 vs. initial pitch angle.

Table 10 compares the maximum force of the primary strut when initial pitch angles are $0^{\circ}, 6.81^{\circ}$ (maximum force angle of primary strut 2), and $20^{\circ}$ (maximum force angle of primary strut 1 ). When the initial pitch angle is 6.81 , the maximum force of the primary strut $1 / 2$ is $3.98 \times 10^{4} \mathrm{~N} / 4.1$ $\times 10^{4} \mathrm{~N}$, respectively, and the error is $7.5 \% / 10.8 \%$. When the initial pitch angle is 20 , the maximum force on primary strut $1 / 2$ is $4.22 \times 10^{4} \mathrm{~N} / 3.6 \times 10^{4} \mathrm{~N}$, respectively, and the error with the initial pitch angle of $0^{\circ}$ is $14 \% /-2.7 \%$, respectively. It can be seen that the initial pitch angle has a certain impact on the maximum force of the primary strut, and the maximum force of the primary strut 1 and the primary strut 2 is substantially equal.

4.4. Impact on the Secondary Strut by Initial Pitch Angle. Figure 16 shows the relationship between the maximum force of the secondary strut 1 and the initial pitch angle. It can be seen from the figure that when the initial pitch angle is 0 , the maximum force of the secondary strut 1 is $2.7 \times$ $10^{4} \mathrm{~N}$. When the initial pitch angle falls in range of $0 \sim 0.11 \mathrm{rad}$, the maximum force of the secondary strut 1 decreases when the initial pitch angle increases. When the pitch angle is $0.11 \mathrm{rad}$, the maximum force of the secondary strut 1 is $1.4 \times 10^{4} \mathrm{~N}$; when the initial pitch angle is greater than $0.11 \mathrm{rad}$, the maximum force of the secondary strut 1 increases when the initial pitch angle increases also. When the initial pitch angle is $0.34 \mathrm{rad}$, the maximum force of the secondary strut 1 is $2.6 \times 10^{4} \mathrm{~N}$.

Figure 17 shows a graph showing the relationship between the maximum force of the secondary strut 2 and the initial pitch angle. It can be seen from the figure that when the initial pitch angle is 0 , the minimum force of the secondary strut 2 is $2.75 \times 10^{4} \mathrm{~N}$. When the initial pitch angle is $0 \sim 0.11 \mathrm{rad}$, the maximum force of the secondary strut 1 increases when the initial pitch angle increases. When the initial pitch angle is $0.11 \mathrm{rad}$, the maximum value of maximum force of the secondary strut 1 is $4.4 \times 10^{4} \mathrm{~N}$. When the initial pitch angle is greater than $0.11 \mathrm{rad}$, the maximum force of the secondary strut 1 decreases when the initial pitch angle increases. When the initial pitch is $0.34 \mathrm{rad}$, the maximum force on the secondary strut 1 decays to $3.1 \times 10^{4} \mathrm{~N}$.

Table 11 compares the maximum force on the secondary strut at initial pitch angles of $0^{\circ}$ (the maximum force angle of the secondary strut 1 ), $6.87^{\circ}$ (the maximum force angle of the secondary strut 2 ), and $20^{\circ}$. When the initial pitch angle is 6.87 , the maximum force of the secondary strut $1 / 2$ is $1.4 \times 10^{4} \mathrm{~N} / 4.4 \times 10^{4} \mathrm{~N}$, respectively, and the error to $0^{\circ}$ initial pitch angle is $-48.1 \% / 63 \%$, respectively. When the initial pitch angle is $20^{\circ}$, the maximum force on the secondary strut $1 / 2$ is $2.6 \times 10^{4} \mathrm{~N} / 3.2 \times 10^{4} \mathrm{~N}$, and the error to $0^{\circ}$ initial pitch angle is $-3.7 \% / 18.5 \%$, respectively. It can be seen that the initial pitch angle has a significant impact on the maximum force of the secondary strut $1 / 2$. Furthermore, the maximum force of the secondary strut 1 is smaller than that of the secondary strut 2.

According to the analysis above, the impact of the initial pitch angle on the soft-landing performance can be concluded as follows:

(1) The initial pitch angle has less impact on the minimum displacement from the lunar surface

(2) The initial pitch angle has a significant impact on the overload of the body, which can also be considered to effectively reduce the overload of the body

(3) The initial pitch angle has a certain impact on the maximum force of the primary strut, and the maximum force on the primary strut 1 and the primary strut 2 is substantially equal

(4) The initial pitch angle has a great impact on the maximum force of the secondary strut $1 / 2$, and the maximum force of the secondary strut 1 is smaller than that of the secondary strut 2 
TABLE 10: Impact of typical working conditions of initial pitch angle on maximum force of primary strut.

\begin{tabular}{lccc}
\hline Initial pitch angle & Primary strut $1 / 2$ max force $(\mathrm{N})$ & Error & Note \\
\hline $0^{\circ}$ & $3.7 \times 10^{4 / 3.7 \times 10^{4}}$ & $/$ & Maximum force on primary strut 1 \\
$20^{\circ}(\pi / 9)$ & $4.22 \times 10^{4 / 3} 3.6 \times 10^{4}$ & $14 \% /-2.7 \%$ & Maximum force on primary strut 2 \\
$6.87^{\circ}(0.12)$ & $3.98 \times 10^{4 /} 4.1 \times 10^{4}$ & $7.5 \% / 10.8 \%$ & . \\
\hline
\end{tabular}

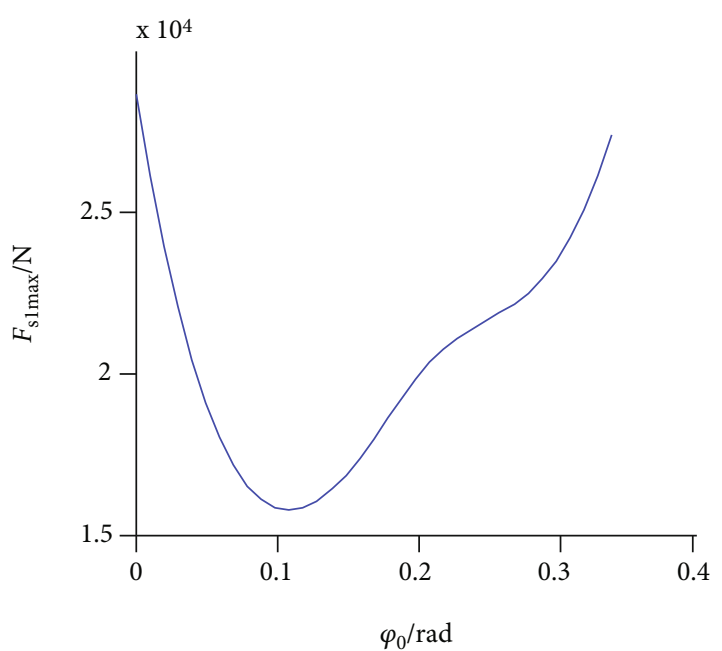

FIGURE 16: Max force on the secondary strut 1 vs. initial pitch angle.

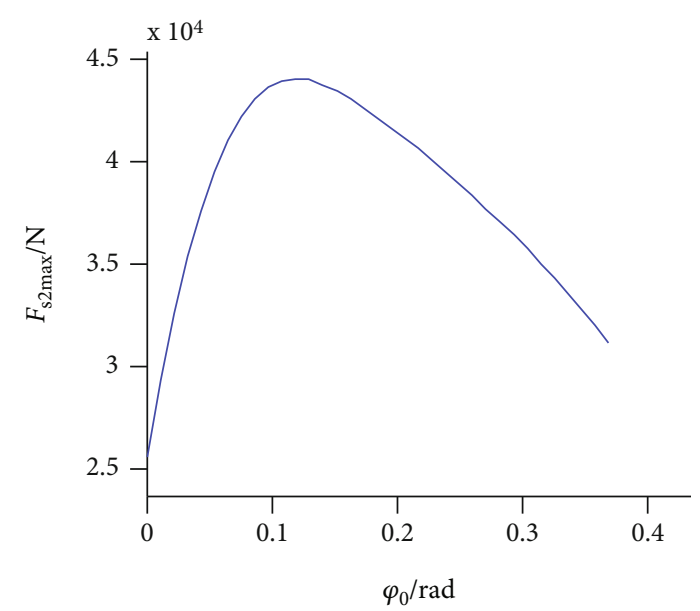

Figure 17: Max force on the secondary strut 2 vs. initial pitch angle.

\section{The Impact of Lunar Inclination on Soft- Landing Performance}

The environment of the lunar landing is relatively rigor in most cases. The lunar landing field can be categorized into two types without considering other topographical factors. One is a horizontal lunar surface, and the other is a lunar slope with certain inclination. When the lander lands on the lunar slope, the surface inclination will have impact on the soft landing. An excessive lunar surface inclination may make the lander lose stability and even overturn to fail the entire mission. Therefore, it is extremely necessary and significant to study and analyze the impact of the lunar inclination on the soft-landing performance of the lunar lander. Table 12 shows the simulation analysis conditions of the lunar lander soft landing.

5.1. Impact on Body Displacement by Lunar Inclination. Figure 18 shows the change of body displacement by time under different lunar surface inclinations. It can be seen from the figure that when the lunar surface inclination is not 0 , the body displacement change can be divided into 3 stages: In the initial stage, after the foot pad 1 touches the ground, the body rotates around the foot pad 1 until the foot pad 2 touches the ground. In the second stage, after the foot pads 1 and 2 are grounded, the body displacement changes. In the third stage, the foot pad 1 leaves the lunar surface again. This scenario will only occur after the lunar surface inclination is greater than a certain value. It can be seen from Figure 18 that when the lunar surface inclination is greater than $15^{\circ}(\pi / 12)$, the body displacement change has an obvious rebound, which indicates that the foot pad 1 has left the lunar surface again. As the lunar inclination increases, the body displacement changes more drastically. When the lunar inclination exceeds a certain angle, the body will roll and overturn around the foot pad 2. Figure 19 is the diagram between the minimum displacement of the body from the lunar surface and the lunar surface inclination. It can be seen from the figure that the minimum distance between the body and the lunar surface decreases with the increase of the lunar surface inclination, which can be approximately considered as a linear decreasing relationship. From Figures 18 and 19, it can be seen that when the lander lands on the lunar slope, the foot pad 1 should be prevented from leaving the lunar surface again to avoid rollover and overturning. Therefore, when choosing a landing field, a certain safety factor should be considered. It is recommended that the inclination of the lunar slope for landing should not be greater than $9^{\circ}(\pi / 20)$.

Table 13 compares the minimum distance between the body and the lunar surface when landing on the horizontal terrain of the lunar surface and the slope terrain. The error of the lunar surface inclination $\theta=0^{\circ}$ and $\theta=27.7^{\circ}(\pi / 6.5)$ is $-50 \%$. It can be seen that landing on a slope terrain has a greater impact on the minimum distance between the body and the lunar surface. 
TABLE 11: Impact of typical working conditions of initial pitch angle on the maximum force of secondary strut.

\begin{tabular}{lccc}
\hline Initial pitch angle & Max force on secondary strut $1 / 2(\mathrm{~N})$ & Error & Note \\
\hline $0^{\circ}$ & $2.7 \times 10^{4} / 2.7 \times 10^{4}$ & $/$ & Max force on secondary strut 1 \\
$20^{\circ}(\pi / 9)$ & $2.6 \times 10^{4} / 3.2 \times 10^{4}$ & $-3.7 \% / 18.5 \%$ & $/$ \\
$6.87^{\circ}(0.12)$ & $1.4 \times 10^{4} / 4.4 \times 10^{4}$ & $-48.1 \% / 63 \%$ & Max force on secondary strut 2 \\
\hline
\end{tabular}

TABLE 12: Simulation analysis conditions of soft landing.

\begin{tabular}{lccccccc}
\hline $\begin{array}{l}\text { Thrown mass } \\
(\mathrm{kg})\end{array}$ & $\begin{array}{c}\text { Landing } \\
\text { mode }\end{array}$ & $\begin{array}{c}\text { Vertical speed } \\
(\mathrm{m} / \mathrm{s})\end{array}$ & $\begin{array}{c}\text { Horizontal speed } \\
(\mathrm{m} / \mathrm{s})\end{array}$ & $\begin{array}{c}\text { Initial pitch } \\
\text { angle }\left(^{\circ}\right)\end{array}$ & $\begin{array}{c}\text { Landing strut touchdown } \\
\text { sequence }\end{array}$ & $\begin{array}{c}\text { Lunar soil } \\
\text { state }\end{array}$ & $\begin{array}{c}\text { Gravity } \\
\text { field }\end{array}$ \\
\hline 1350 & $2-2$ & 4.0 & 0 & 0 & $2 / 3-1 / 4$ & Flexible & Moon \\
\hline
\end{tabular}

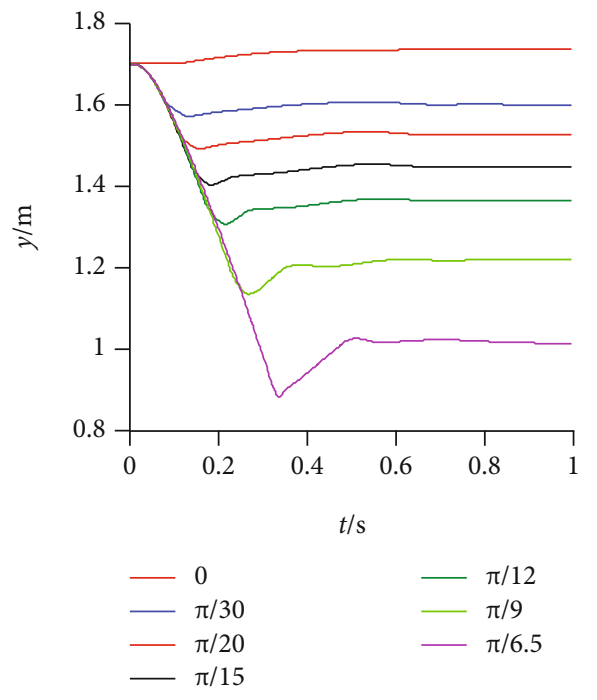

Figure 18: Body displacement vs. time.

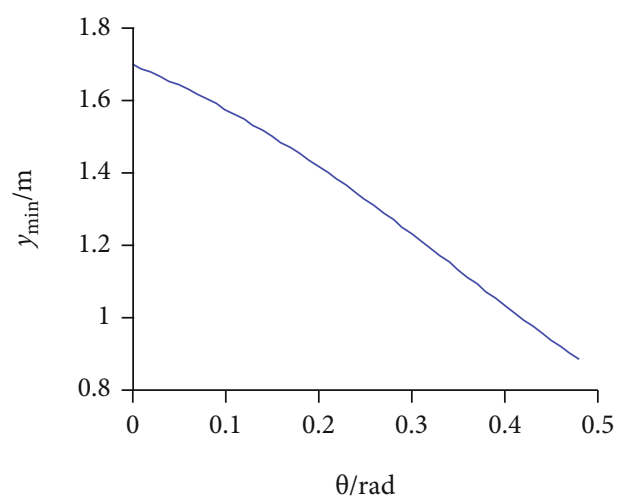

FIGURE 19: Minimum displacement of the body from the lunar surface vs. the lunar surface inclination.

5.2. Impact on Body Overload by Lunar Inclination. Figure 20 shows the body's overload at different lunar inclinations in time domain. It can be seen from the figure that when the lander lands on the horizontal lunar surface, the peak is about $6.8 g_{n}$ which is greater than the overload peak when it lands on the inclined lunar surface. When the lander lands on the lunar slope with different inclinations, there are at least two overload peaks. Based on the analysis, this is because the two pads touch the ground at different times. The magnitude and time of the first overload peak are basically the same. As the lunar surface inclination increases, the time interval between the second overload peak and the first overload peak also increases. It is analyzed that as the inclination of the lunar surface increases, the time for the body to rotate around the foot pad 1 is also longer. When the lunar inclination is greater than $15^{\circ}(\pi / 12)$, the third overload peak appears, which indicates that the body has rotated around the foot pad 2, and the foot pad 1 has left the lunar surface again. Figure 21 shows the curve of the maximum overload of the body with the change of the lunar surface inclination. It can be seen that the lunar inclination can reduce the maximum overload of the body. When the inclination of the lunar slope is less than $9^{\circ}(\pi / 20)$, the maximum overload of the body decreases linearly with the inclination of the lunar slope, and the maximum overload of the body during landing on the horizontal lunar surface is reduced from $6.8 g_{n}$ to $4 g_{n}$ (maximum body overload at $9^{\circ}(\pi / 20)$ with lunar inclination being $\left.9^{\circ}(\pi / 20)\right)$. When the inclination of the lunar slope is between $9^{\circ}(\pi / 20)$ and $20^{\circ}(\pi / 9)$, the maximum overload of the lunar lander stays at $4 g_{n}$ which is basically unchanged. When the inclination of the lunar slope is greater than $20^{\circ}(\pi / 9)$, the maximum overload of the lunar lander slowly decreases again.

Table 14 compares the maximum overload of the body when landing on the horizontal lunar terrain and the slope terrain. When the lunar inclination angles are $\theta=0^{\circ}$ and $\theta$ $=27.7^{\circ}(\pi / 6.5)$, the error is $-55.9 \%$. It can be seen that landing on a slope terrain has a greater impact on the body maximum overload.

5.3. Impact on the Primary Strut by Lunar Inclination. Figure 22 shows the relationship between the maximum force on the primary strut 1 and the inclination of the lunar surface. The maximum force on the primary strut 1 decreases with the increase of the lunar inclination. The maximum force of the primary strut 1 is $3.7 \times 10^{4} \mathrm{~N}$ when the lunar inclination is $0^{\circ}$, and the maximum force of the 
TABLE 13: Impact on the minimum body displacement under typical lunar inclination conditions.

\begin{tabular}{lcc}
\hline Lunar inclination $\left(^{\circ}\right)$ & Minimum body displacement $(\mathrm{m})$ & Error $*$ \\
\hline 0 & 1.7 & $/$ \\
$27.7(\pi / 6.5)$ & 0.85 & $-50 \%$ \\
\hline
\end{tabular}

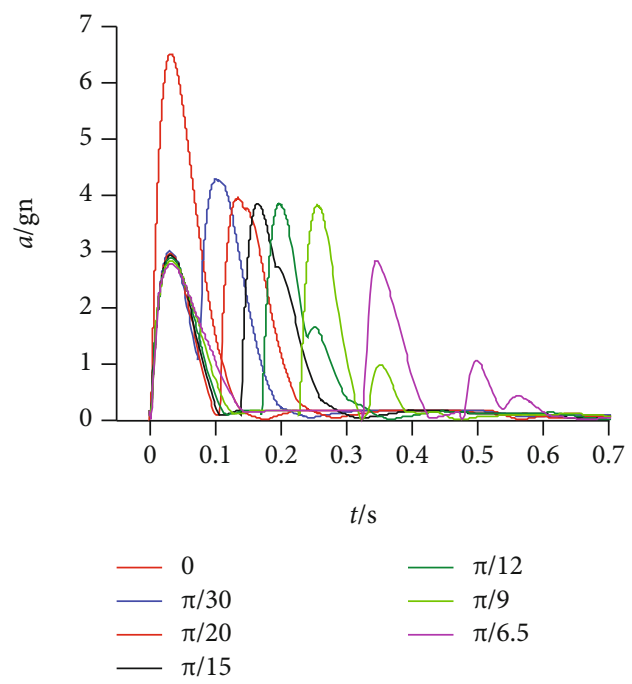

FIgURE 20: Body overload vs. time.

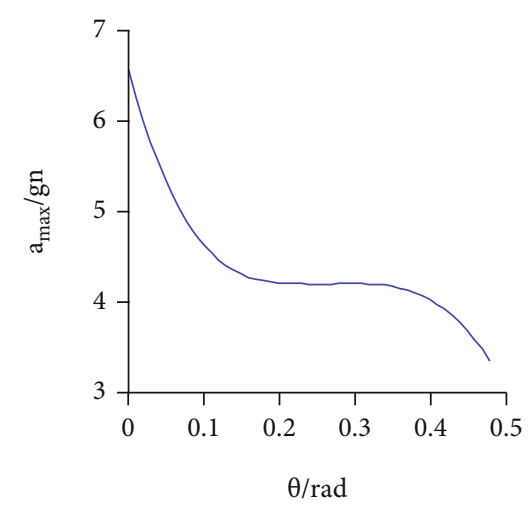

Figure 21: The maximum overload of the body with the lunar inclination.

TABLE 14: Impact on the maximum body overload under typical lunar inclination conditions.

\begin{tabular}{lcc}
\hline Lunar inclination $\left(^{\circ}\right)$ & Body maximum overload $\left(g_{n}\right)$ & Error* \\
\hline 0 & $6.8 g_{n}$ & $/$ \\
$9(\pi / 20)$ & $4 g_{n}$ & $-41.2 \%$ \\
$27.7(\pi / 6.5)$ & $3 g_{n}$ & $-55.9 \%$ \\
\hline
\end{tabular}

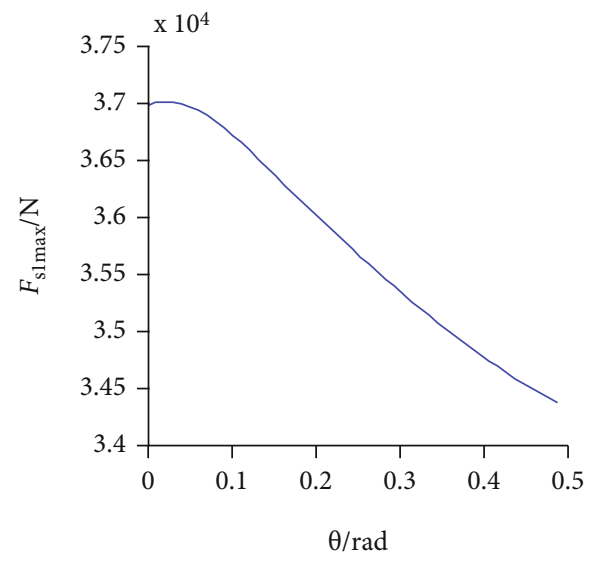

FIgURE 22: The maximum force of the primary strut 1 varies with the lunar inclination.

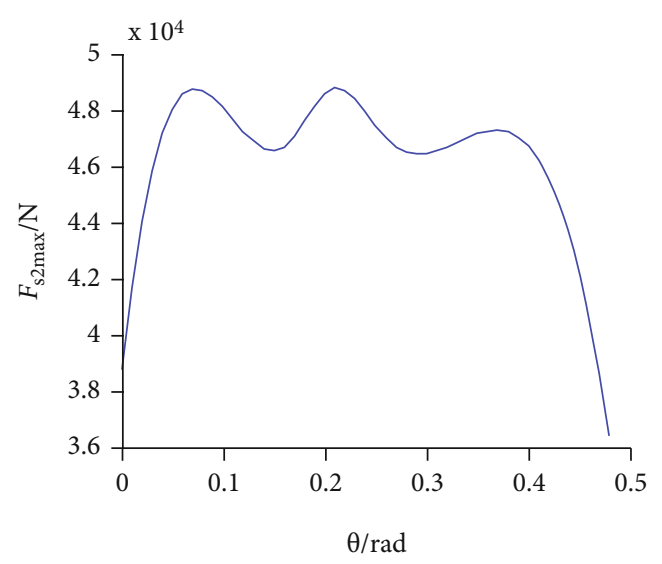

FIgURE 23: The maximum force of the primary strut 2 varies with the lunar inclination.

primary strut 1 is $3.42 \times 10^{4} \mathrm{~N}$ when the lunar inclination angle is $\pi / 6.5$. Figure 23 shows the relationship between the maximum force of the primary strut 2 and the inclination of the lunar slope. When the lunar inclination angle is $0^{\circ}$, the maximum force of the primary strut 2 is $3.9 \times 10^{4} \mathrm{~N}$ . The peak time of the primary strut 2 increases with the increase of the lunar inclination. The maximum force of the primary strut 2 with the lunar slope at $\pi / 30-\pi / 9$ is between $4.6 \times 10^{4} \mathrm{~N}$ and $4.9 \times 10^{4} \mathrm{~N}$. When the inclination angle of the lunar surface slope is at $\pi / 6.5$, the maximum force of the primary strut 2 is reduced to $3.6 \times 10^{4} \mathrm{~N}$.

Table 15 compares the maximum force of primary struts 1 and 2 when landing on the lunar horizontal terrain and slope terrain. When the lunar inclination is $\theta=0^{\circ}$, the maximum forces of primary strut 1 and primary strut 2 are 3.7 $\times 10^{4} \mathrm{~N}$ and $3.9 \times 10^{4} \mathrm{~N}$, respectively. When the lunar inclination is $\theta=27.7^{\circ}(\pi / 6.5)$, the maximum forces of the primary strut 1 and the primary strut 2 are $3.42 \times 10^{4} \mathrm{~N}$ and $3.6 \times 10^{4} \mathrm{~N}$, respectively. The maximum force errors of the 
TABLE 15: Impact on the maximum force of the primary strut under typical lunar inclination conditions.

\begin{tabular}{lccc}
\hline Lunar inclination $\left(^{\circ}\right)$ & Primary struts $1 / 2$ max force $(\mathrm{N})$ & Error $*$ & Note \\
\hline 0 & $3.7 \times 10^{4} / 3.9 \times 10^{4}$ & $/$ & The primary strut 1 has the greatest force \\
$12(\pi / 15)$ & $3.6 \times 10^{4} / 4.9 \times 10^{4}$ & $-2.7 \% / 25.6 \%$ & The primary strut 1 has the greatest force \\
$27.7(\pi / 6.5)$ & $3.42 \times 10^{4} / 3.6 \times 10^{4}$ & $-7.6 \% /-7.7 \%$ & Both primary struts 1 and 2 have the least force \\
\hline
\end{tabular}

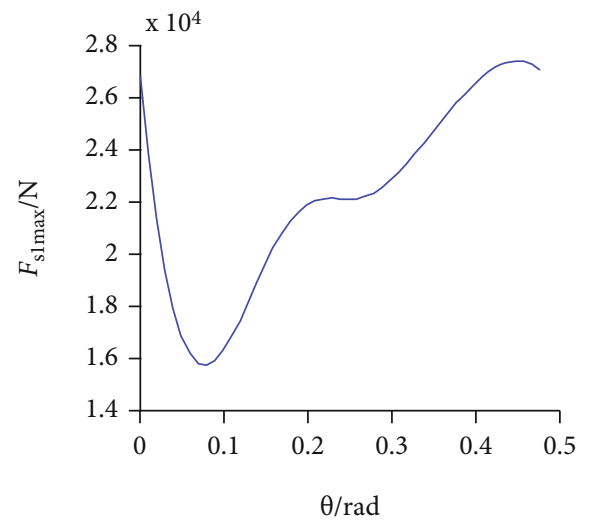

FIGURE 24: The maximum force of the secondary strut 1 varies with the lunar inclination.

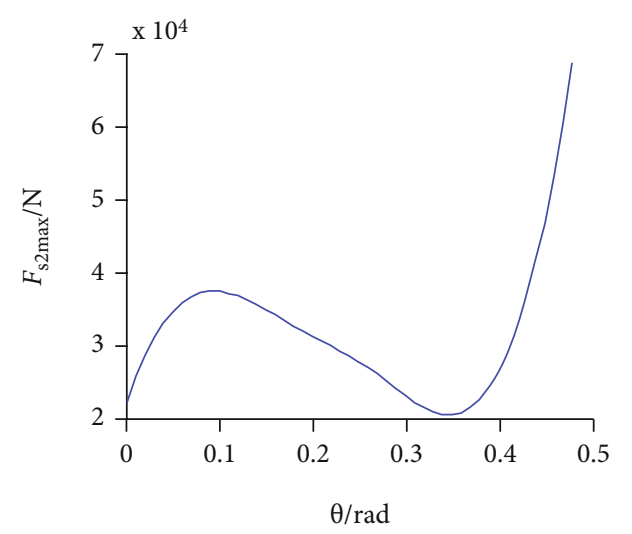

Figure 25: The maximum force of the secondary strut 2 varies with the lunar inclination.

two primary struts are $-7.6 \%$ and $-7.7 \%$ compared to $0^{\circ}$ lunar inclination condition. When the lunar inclination is $\theta=12^{\circ}$ $(\pi / 15)$, the maximum forces of the primary strut 1 and the primary strut 2 are $3.6 \times 10^{4} \mathrm{~N}$ and $4.9 \times 10^{4} \mathrm{~N}$, respectively, and the maximum force errors of the two primary struts are $-2.7 \%$ and $25.6 \%$ compared to the $0^{\circ}$ lunar inclination condition. It can be seen that the lunar landing on slope terrain has little effect on the maximum force of the primary strut.

5.4. Impact on the Secondary Strut by Lunar Inclination. Figure 24 shows the relationship between the maximum force on secondary strut 1 and the inclination of the lunar slope. The maximum force of secondary strut 1 decreases rapidly when the lunar inclination is between 0 and $\pi / 30$, and its minimum value is $1.58 \times 10^{4} \mathrm{~N}$. When the inclination of the lunar surface is $\pi / 30 \sim \pi / 15$, the maximum force of the secondary strut 1 increases with the increase of the inclination of the lunar surface. When the lunar inclination angle is $\pi / 15 \sim \pi / 12$, the maximum force of secondary strut 1 does not change with the lunar inclination, but is a constant value, which is $2.2 \times 10^{4} \mathrm{~N}$. When the lunar inclination is greater than $\pi / 12$, the maximum force on the secondary strut 1 increases again with the increase in the lunar inclination, and the maximum value is $2.8 \times 10^{4} \mathrm{~N}$. Figure 25 shows the relationship between the maximum force value on the secondary strut 2 and the inclination of the lunar slope. In the range of 0 to $\pi / 30$ of the lunar inclination, the maximum force of the secondary strut 2 has an increasing relationship with the lunar inclination, and its maximum value is $4.1 \times$ $10^{4} \mathrm{~N}$. When the lunar inclination is $\pi / 30 \sim \pi / 9$, the maximum force of secondary strut 2 decreases with the increase of lunar inclination, and its minimum value is $2.1 \times 10^{4} \mathrm{~N}$. When the lunar inclination is $\pi / 9 \sim \pi / 6$, the maximum force of secondary strut 2 increases rapidly with the increase of the lunar inclination again, and the maximum is $6.85 \times 10^{4} \mathrm{~N}$.

Table 16 compares the maximum forces of secondary struts 1 and 2 when landing on the lunar horizontal terrain and slope terrain. When the lunar inclination is $\theta=0^{\circ}$, the maximum forces of secondary strut 1 and secondary strut 2 are $2.7 \times 10^{4} \mathrm{~N}$ and $2.6 \times 10^{4} \mathrm{~N}$, respectively. When the lunar inclination is $\theta=27.7^{\circ}(\pi / 6.5)$, the maximum forces of the secondary strut 1 and the secondary strut 2 are 2.8 $\times 10^{4} \mathrm{~N}$ and $6.85 \times 10^{4} \mathrm{~N}$, respectively, and the maximum force errors of the two struts are $3.7 \%$ and $153 \%$ compared to $0^{\circ}$ inclination condition. When the lunar inclination angle is $\theta=6^{\circ}(\pi / 30)$. The maximum forces of the secondary strut 1 and the secondary strut 2 are $1.58 \times 10^{4} \mathrm{~N}$ and $4.1 \times 10^{4} \mathrm{~N}$, respectively, and the maximum force errors of the two struts are $-41.5 \%$ and $57.7 \%$ compared to the $0^{\circ}$ inclination condition. It can be seen that the lunar landing on a slope terrain has a certain impact on the maximum force on secondary strut 1 and has a great impact on the maximum force on secondary strut 2 .

According to the analysis above, the impact on softlanding performance by lunar inclination can be concluded as follows:

(1) The inclination of the lunar slope has a great impact on the minimum distance between the body and the lunar surface

(2) The inclination of the lunar slope has a great impact on the body overload, and the landing along the lunar slope will effectively reduce the body overload 
TABLE 16: Impact on the maximum force of secondary struts under typical lunar inclination conditions.

\begin{tabular}{lccc}
\hline Lunar inclination $\left(^{\circ}\right)$ & Secondary struts $1 / 2$ max force $(\mathrm{N})$ & Error $*$ & Note \\
\hline 0 & $2.7 \times 10^{4} / 2.6 \times 10^{4}$ & $/$ & $/$ \\
$6(\pi / 30)$ & $1.58 \times 10^{4} / 4.1 \times 10^{4}$ & $-41.5 \% / 57.7 \%$ & The secondary strut 1 has the least force \\
$27.7(\pi / 6.5)$ & $2.8 \times 10^{4} / 6.58 \times 10^{4}$ & $3.7 \% / 153 \%$ & Both secondary struts 1 and 2 have the maximum force \\
\hline
\end{tabular}

(3) The inclination of the lunar slope has little impact on the maximum force of the primary strut

(4) The inclination of the lunar slope has a great impact on the maximum force of secondary strut 1 and significant impact on the maximum force on secondary strut 2

(5) According to the analysis above and comprehensive consideration, it is recommended that, when the lander lands along the lunar slope, the inclination of the lunar slope should not be greater than $9^{\circ}$

\section{Conclusions}

In this paper, the impacts on the soft-landing dynamics in 2-2 landing mode are analyzed in terms of two initial landing conditions which include horizontal landing velocity and pitch angle of lunar lander using 7 DOF soft-landing dynamics models of the lunar lander:

(1) The maximum error influences of the initial horizontal velocity on the minimum distance from the lunar surface, the body overload, the force on the primary strut, and the force on the secondary strut are $2.36 \%, 36.8 \%, 52.5 \%$, and $313.3 \%$, respectively. It can be seen that the initial horizontal velocity has a great impact on the maximum forces of the primary strut and secondary strut

(2) The maximum error influences of the initial pitch angle on the minimum distance from the moon surface, the body overload, the force on the primary strut, and the force on the secondary strut are $9.6 \%, 49.2 \%, 14 \%$, and $63 \%$, respectively. It can be seen that the initial pitch angle has a great impact on the body overload and the maximum forces on the secondary strut

(3) The impacts of the lunar inclination on the minimum body distance from the lunar surface, the body overload, and the maximum errors of the primary strut force and the secondary strut force are $50 \%$, $55.9 \%, 25.6 \%$, and $153 \%$, respectively. It can be seen that the inclination of the lunar slope has a great impact on the minimum body distance from the lunar surface, the body overload, and the maximum force on the secondary strut

(4) The body overload and landing impact could be significantly mitigated if the lunar probe's horizontal landing speed is limited within $1 \mathrm{~m} / \mathrm{s}$, the pitch angle is limited within $12^{\circ}$, and the landing is along the uphill terrain with the inclination of the lunar slope less and equal to $9^{\circ}$

\section{Data Availability}

The data used to support the findings in this investigation is included within the manuscript.

\section{Conflicts of Interest}

The authors declare that they have no conflicts of interest.

\section{Acknowledgments}

This work was sponsored by the Natural Science Foundation of Shanghai (No. 16ZR1436100).

\section{References}

[1] H. H. Doiron and G. A. Zupp, Apollo Lunar Module Landing Dynamics. AIAA-2000-1678, InDyne, Inc., 2000.

[2] R. E. Lavender, Equations for Two-Dimensional Analysis of Touchdown Dynamics of Spacecraft with Hinged Legs Including Elastic, Damping, and Crushing Effects, vol. 66, article 23668, George C. Marshall Space Flight Center N, Huntsville, 1963.

[3] R. E. Lavender, Touchdown Dynamics Analysis of Spacecraft for Soft Lunar Landing, George C. Marshall Space Flight Center NASA TN D-2001, Huntsville, 1964.

[4] L. Robert, On Touchdown Dynamics Analysis for Lunar Landing, American Institute of Aeronautics and Astronautics, 1965.

[5] R. J. Black, "Quadrupedal landing gear systems for spacecraft," Journal of Spacecraft and Rockets, vol. 1, pp. 196-203, 1964.

[6] W. C. Walton, B. J. Durling, and H. W. Leonard, Application of Digital Computer Techniques to the Study of the Impact Dynamics of Lunar-Landing Vehicles, Langley Research Center N 66-15238, Hampton, 1964.

[7] W. C. Walton, R. W. Herr, and H. W. Leonard, "Studies of touchdown stability for lunar landing vehicles," Journal of Spacecraft and Rockets, vol. 1, no. 5, pp. 552-556, 1964.

[8] H. H. Doiron and G. A. Zupp, A Mathematical Procedure for Predicting the Touchdown Dynamics of a Soft-Landing Vehicle, Manned Spacecraft Center NASA TN D-7045, Houston, 1971.

[9] J. L. Wan, H. Nie, and L. C. Li, “Analysis of the landing impact performance for lunar landing leg with transient dynamic method," Acta Armamentarii, vol. 31, pp. 22-28, 2010.

[10] Q. Lin and H. Nie, "Soft landing impact simulation of lunar lander," Chinese Space Science and Technology, vol. 31, pp. 70-75, 2011.

[11] Q. Lin, Z. Kang, J. Ren, Q. Zhao, and H. Nie, "Impact analysis of lunar lander soft landing performance caused by the body gravity centerline shift," Journal of Aerospace Engineering, vol. 28,2015 